\title{
DESAFÍOS DE LA TELEVISIÓN ANTE LA CONSOLIDACIÓN DEL ECOSISTEMA DIGITAL
}

\author{
Marta Roel \\ (Universidad de Murcia) \\ mroel@um.es
}

\begin{abstract}
Resumen: La generalización de la tecnología digital está llevando al sector televisivo a experimentar numerosas transformaciones y a afrontar el paso de la Neotelevisión a la Postelevisión, una nueva edad televisiva caracterizada por la abundancia y personalización de la oferta, la convergencia y la interactividad. En este proceso de consolidación del ecosistema digital, el sector de la televisión debe ser capaz de afrontar y dar respuesta -al menos- a cinco desafíos tal y como se refleja en el presente artículo.
\end{abstract}

Palabras clave: Postelevisión, apagón analógico, España.

Abstract: Generalization of digital technology is taking the television sector to experience many changes and address the transition from the Neotelevision to the Postelevision, a new television age characterized by the abundance, offering customization, convergence and interactivity.In this process of consolidation of the digital ecosystem, the television should be able to deal with and respond - at least - five challenges as reflected in this article.

Keywords: Postelevision, switch off, Spain.

\section{INTRODUCCIÓN}

n la Unión Europea vivimos una etapa de cuenta atrás para proceder al apagón analógico'. En España los primeros apagados analógicos comenzaron a realizarse a partir de junio de 2009. Este proceso culminó el 2 de abril de 2010, un día antes de la fecha límite establecida para el cese de las emisiones en analógico (Impulsa TDT, 2010). Pero éste es sólo un primer paso -tecnológico- hacia la progresiva consolidación del ecosistema audiovisual digital. A lo largo de este proceso, irá echando raíces el Digital Lifestyle con sus rasgos característicos sistematizados a través de la denominada Triple $\boldsymbol{A}$ que hace referencia a tres vocablos anglosajones -Anywhere, Anytime y Anyway ${ }^{2}-$

\footnotetext{
${ }^{1}$ Algunos países europeos ya han procedido a realizar el apagón analógico. Es el caso de Luxemburgo y Holanda en 2006; de Finlandia y Suecia en 2007; y de Suiza y Alemania en 2008. En 2010 se ha completado en Bélgica y España y si se cumplen las previsiones, antes de que finalice el año se sumarán Austria, Letonia, Estonia, Croacia, Eslovenia y Malta (Impulsa TDT, 2010).

${ }^{2}$ En cualquier lugar (Anywhere), en cualquier momento (Anytime) y de todos modos, a través de diversas plataformas (Anyway).
} 
que resumen esquemáticamente los atributos de una nueva edad televisiva denominada por algunos autores como Postelevisión ${ }^{3}$ (Piscitelli, 1998; Ramonet, 2002; Imbert, 2008; Roel, 2008).

La Postelevisión y su singular estilo de vida digital, dota al usuario de una mayor movilidad y autonomía en el consumo de contenidos audiovisuales. La recepción ya no tiene por qué realizarse desde los hogares, dado que se permite el acceso a los contenidos elegidos en cualquier momento, en el instante que se decida. La tecnología posibilita el consumo bajo demanda y, además, el usuario puede beneficiarse de las diversas formas de difusión de los contenidos, optando por pantallas alternativas (teléfono móvil, PDA, PC, etc.). Todos estos nuevos hábitos de consumo audiovisual multipantalla contribuyen a crear y consolidar el moderno estilo de vida digital.

La Postelevisión, caracterizada por la abundancia y la personalización de la oferta, la convergencia y la interactividad, supone desde el punto de vista de la gestión de contenidos una revolución, una ruptura con las formas tradicionales de creación, difusión y recepción audiovisual e implica la necesidad de diseñar nuevas políticas públicas que sean capaces de dar respuesta a la evolución del sector.

Tomando como referencia este contexto de digitalización de la televisión, la explosión de las diversas modalidades de distribución de los contenidos televisivos (TDT, satélite, cable, IPTV, televisión en movilidad), la diversificación de las plataformas de recepción (televisor, ordenador personal, dispositivos móviles) y la especial relevancia que adquieren los usuarios en este nuevo escenario, se ha procedido a delimitar cinco desafíos de especial relevancia que el sector de la televisión debe ser capaz de afrontar en ese camino que se ha iniciado hacia la consolidación de la Postelevisión: la democratización digital, la adecuación de los contenidos audiovisuales al nuevo entorno tecnológico, la búsqueda de nuevas fórmulas de financiación ante la evidencia del agotamiento del modelo tradicional, la apuesta por la calidad, aunque ya no sea posible mantener por mucho tiempo el consumo masivo impulsado por las tradicionales ofertas lineales, y la protección de los menores.

La delimitación de estos desafíos obedece, en esencia, a la reflexión personal de la autora que tras años de investigación sobre la materia y observación del sector audiovisual ha detectado como determinantes en la naciente etapa de digitalización de la televisión. Para afrontar este estudio, se ha procedido a realizar una amplia revisión y actualización bibliográfica y hemerográfica que ha permitido avalar los planteamientos de partida, formulando una serie de propuestas y conclusiones, tal y como se explicita a continuación.

\footnotetext{
${ }^{3}$ Paleotelevisión y Neotelevisión son las edades que preceden a la Postelevisión. En Roel (2006) se puede obtener una explicación detallada sobre ambas etapas.
} 


\section{LA DEMOCRATIZACIÓN DIGITAL}

La revolución digital ha traído consigo diversas innovaciones que han repercutido positivamente sobre el sector audiovisual en su conjunto: la optimización del ancho de banda disponible, que posibilita la explosión de la oferta, el incremento en la calidad de imagen y sonido que permite a los usuarios beneficiarse de una televisión sin ruidos ni interferencias, o la interactividad que propicia la consolidación de un nuevo estatus para el usuario, transformando la unidireccionalidad tradicional de la comunicación a través de la televisión en bidireccionalidad, pasando de este modo a ostentar un papel aún más destacado en el proceso comunicativo.

Además, la implantación de la tecnología digital ha favorecido la aparición y desarrollo de distintas modalidades de distribución de la señal televisiva (TDT, Satélite, Cable, ADSL), pero también están experimentando un importante despegue otras fórmulas que tradicionalmente no estaban vinculadas al sector, es el caso de Internet y de los dispositivos móviles, tal y como se avanzaba anteriormente.

Efectivamente, la digitalización del sector audiovisual se ha hecho realidad en diversos países, también en España. Ahora, es preciso detectar el nivel de implantación real de la tecnología digital en el tejido social, con objeto de proponer líneas de desarrollo efectivas capaces de contribuir positivamente en el proceso de consolidación de la Postelevisión.

Los últimos datos disponibles ponen de manifiesto que en España el $44,6 \%$ de los hogares disponen de banda ancha y el $30 \%$ del parque de terminales móviles disponen de capacidad 3G (Fundación Telefónica, 2009). Ello nos permite deducir que el camino hacia esa deseable sociedad hiperconectada, ubicua, con conexión a Internet permanente, se ha iniciado; avanza con paso firme pero aún está lejos de ser una realidad de uso común para el conjunto de la población. De hecho, la actual crisis económica que afronta el Estado español mientras se escriben estas líneas, podría agravar el proceso de generalización de las TIC.

Coincidimos con Bustamante (2007) en sus planteamientos en torno a la necesidad de una reorientación de los planes de la Sociedad de la Información (S.I.), de modo que se posibilite un acceso a las redes más barato y universal. De lo contrario, las nuevas redes digitales podrían verse como un nuevo espacio público de carácter elitista y desigual capaces de fragmentar interna y globalmente el mundo (Zallo, 2007).

Respecto a la implantación de la tecnología digital en los televisores, los últimos datos a los que hemos tenido acceso ponen de manifiesto que al tiempo que se procedía a completar el apagón analógico, el número de televisores con sintonizador de TDT integrado era de 10,8 millones, mientras que la cifra de decodificadores externos (set top box) era sensiblemente superior, en torno a los 30 millones de unidades en hogares principales. Y tan solo el $8 \%$ del parque de televisores españoles disponía de sintonizador de HD integrado (Impulsa TDT, 2010). 
Estos datos nos llevan a afirmar que si bien se ha producido progresivamente un considerable avance, por el momento las cifras no resultan satisfactorias de cara a la consolidación del ecosistema digital, y por ende, de la Postelevisión.

En la actualidad, prácticamente la totalidad de la población española $(99,8 \%)$ tiene acceso a la TDT pero éste se ha producido a través de un desembolso económico limitado, adquiriendo mayoritariamente un decodificador y no un televisor integrado. Por lo tanto, el potencial acceso a todos los beneficios que proporciona la tecnología digital se prevé insuficiente, por el momento.

Esta limitada penetración del número de televisores integrados puede producir efectos negativos sobre el desarrollo de un mercado de los contenidos audiovisuales digitales innovador. Por el momento, la presencia de la tecnología digital en el sector ha incidido en mayor medida sobre la diversificación de las modalidades de distribución de los contenidos que sobre una innovación real en contenidos, tal y como se pone de manifiesto a continuación.

Numerosos operadores de televisión han diseñado portales en Internet desde los que es posible beneficiarse de la televisión a la carta donde se incluye una selección de programas emitidos por la cadena recientemente o incluso acceder a programación de archivo ${ }^{4}$. Asimismo, están proliferando otras fórmulas de distribución de contenidos audiovisuales a través de Internet. Sirva como ejemplo las impulsadas por aquellos operadores de televisión que emiten exclusivamente a través de Internet (Akimo-EE.UU., por los proveedores de servicios de Internet -Google Video Store- o las impulsadas por los fabricantes de dispositivos (Apple iTunes).

También los dispositivos móviles se han convertido en plataforma capaz de distribuir contenidos audiovisuales. Su estrategia comercial más habitual consiste en ofertar la suscripción, previo pago de una cuota mensual, a un paquete de canales de televisión ${ }^{5}$.

El desarrollo tecnológico sigue su curso hacia la consolidación del ecosistema digital. Ahora es preciso que la tecnología esté al alcance de la mayor parte de la población, garantizando, por un lado, áreas amplias de cobertura y, por otro, precios de acceso asequibles. El poder político debe garantizar una implantación real de la tecnología, con objeto de no establecer nuevas brechas sociales. Pero este deseable impulso de las políticas públicas debe ser un primer paso que se desarrolle armónicamente y al tiempo que el mercado de los contenidos audiovisuales digitales. Sólo así se conseguirá garantizar las condiciones necesarias para que el sector audiovisual experimente el desarrollo deseado.

\footnotetext{
${ }^{4}$ Es el caso de Radiotelevisión Española (www.rtve.es).

${ }^{5}$ Sirva como ejemplo la oferta en España de Vodafone Live que previo pago de seis euros al mes permite acceder a más de veinticinco canales. Esta oferta se puede consultar en la siguiente dirección electrónica: http://www.live.vodafone.es/TV/vfLiveTVI
} 


\section{LA ADECUACIÓN DE LOS CONTENIDOS AUDIOVISUALES AL NUEVO ENTORNO TECNOLÓGICO}

Tal y como se avanzaba en el epígrafe anterior, la dinamización del entorno digital con la explosión de canales y la consolidación de las distintas plataformas de distribución de contenidos audiovisuales, requiere de la producción de contenidos adecuados a las necesidades del nuevo ecosistema digital. El estratégico sector de los contenidos digitales está llamado a ser el principal vector dinamizador del entorno digital pero, para ello, no sólo es preciso producir mayor volumen de contenidos sino que todos los actores vinculados al sector audiovisual afronten armónicamente y en sintonía una profunda transformación.

El importante despegue tecnológico que ha rediseñado el contexto de la distribución y gestión de contenidos no se refleja de igual modo en el ámbito de la creación de contenidos audiovisuales digitales donde, hasta la fecha, ha existido escasa innovación. En la actualidad, la creación de contenidos se está exportando, mayoritariamente, desde fórmulas tradicionales: lo analógico se transforma en digital, sin más. De modo que no se está dotando a los contenidos de la deseable innovación y valor añadido que técnicamente permite la tecnología digital.

Los operadores/distribuidores apenas asumen riesgos, recurren a fórmulas conocidas, testadas en los mercados audiovisuales que aseguran éxitos comerciales en un escenario dominado mayoritariamente por el consumo masivo de contenidos financiados a través de publicidad. Pero ésta no deja de ser una medida de transición. La estrategia descrita es sólo entendible en un contexto evolutivo, no como parte integradora de un escenario genuinamente digital (Roel, 2009).

Es preciso incentivar la experimentación en narrativas audiovisuales capaces de adaptarse a la nueva realidad creativa que ofrecen las distintas pantallas (televisor, PC, dispositivos móviles) y que ineludiblemente, condicionan estas creaciones culturales. Los procesos de producción de contenidos deben afrontar con creatividad una etapa de experimentación que les permita adecuarse a las nuevas posibilidades narrativas que ofrece la nueva realidad digital.

Aunque técnicamente todos los contenidos se puedan distribuir a través de las distintas pantallas, se deberían respetar parámetros de adecuación a las características singulares de cada una de ellas ya que no todos los contenidos son formalmente aptos para cualquier pantalla. Así, por ejemplo, en el caso de los dispositivos móviles, las reducidas dimensiones de las pantallas así como el contexto de recepción en que previsiblemente se realiza el visionado -ruido ambiente o distracciones externas- son determinantes (García \& Vinader, 2010). La experimentación y creación de contenidos para ser difundidos a través de estos dispositivos debe contar con estos condicionantes. Es recomendable, por tanto, la adecuación entre los parámetros visuales y los soportes emergentes. Pero, por el momento, existe una escasez relativa de producciones solventes con capacidad para alimentar el gigantesco sistema comunicativo (Bustamante, 2007). 
Diversos estudios ponen de manifiesto que los contenidos previsiblemente más valorados serán los informativos, deportes, entretenimiento, business y los contenidos para adultos (De Matteis Tortora, 2006). Los contenidos en directo, difundidos en cápsulas breves de entre uno y tres minutos, con un estilo oral ágil e imágenes impactantes, específicamente pensados para ser consumidos a través de dispositivos móviles. Ya hay experimentos interesantes con informativos realizados en formato modular, ficción seriada en movisodios ${ }^{6}$ o reality shows ${ }^{7}$ dirigidos al mercado de los móviles.

\section{BÚSQUEDA DE NUEVAS FÓRMULAS DE FINANCIACIÓN ANTE LA EVIDENCIA DEL AGOTAMIENTO DEL MODELO TRADICIONAL}

Tradicionalmente la publicidad ${ }^{8}$ ha sido, y sigue siendo, la principal fuente de financiación de la televisión generalista, salvo en el caso de las cadenas públicas que desde sus inicios han contado con importantes sumas procedentes del canon -impuesto por tenencia de televisor- mayoritariamente vigente en los servicios públicos televisivos de nuestro entorno europeo. Si bien, en este aspecto, España constituye una excepción ${ }^{9}$.

\footnotetext{
6"Supervillanos" (2005) es la primera serie española creada para ser distribuida a través de teléfonos móviles de tercera generación. Se trata de una producción de Globomedia compuesta por 40 movisodios de tres minutos cada uno.

${ }^{7}$ El 22 de enero de 2009 se estrenó el reality show español titulado "Bustamente, uno de los nuestros". Se trata de una producción de Endemol Digital pensada para ser distribuida exclusivamente a través de teléfonos móviles e Internet. Integrado por 60 capítulos fragmentados en cápsulas de 6 minutos, ha pretendido dar a conocer aspectos de la vida personal y profesional de David Bustamante, cantante que saltó a la fama tras su aparición en la primera edición de "Operación Triunfo", emitido por TVE 1 entre el 22 de octubre de 2001 y el 11 de febrero de 2002. El formato es innovador pero no es nuevo ya que tres años antes vio la luz "Yo, Melendi" un docu-reality sobre la vida del cantante Ramón Melendi que se emitió en exclusiva a través de la operadora de telefonía móvil Movistar.

${ }^{8}$ Principalmente a través del spot convencional y en menor medida del product placement o la telepromoción.

${ }^{9}$ El canon en España sólo se mantuvo hasta 1965. Fue suprimido por la ley 103/1965 (BOE, 23/12/1965). Desde entonces ha contado con doble financiación -pública y comercial- si bien, ésta última ha ocupado un lugar destacado. Recientemente el modelo de financiación de la televisión pública estatal ha cambiado radicalmente. Algo especialmente notorio desde la entrada en vigor el 1 de enero de 2010 de la Ley 8/2009 de 28 de agosto Corporación RTVE (BOE, 31 de agosto de 2009). Esta ley, impulsada por el gobierno socialista presidido por José Luis Rodríguez Zapatero, establece un mecanismo de financiación que contempla aportaciones del Estado como compensación del servicio público y la comercialización de sus productos y servicios. Pero también establece unas nuevas tasas -aún pendientes del visto bueno de Bruselas- que deberían pagar los operadores de las televisiones comerciales ( $3 \%$ de sus ingresos anuales) y los operadores de telecomunicaciones ( $0,9 \%$ de sus ingresos anuales). Sin ánimo de cuestionar los posibles beneficios que esta medida podría tener sobre la apuesta por la consolidación de la misión de servicio público de la Corporación RTVE, en último término, pretende beneficiar al conjunto de cadenas comerciales que desde el inicio de sus emisiones han acusado a TVE de competencia desleal.
} 
Por el contrario, en el entorno multicanal donde han proliferado las ofertas temáticas se ha optado por la suscripción por acceso a contenidos, el pago por visión (pay per view) o el vídeo bajo demanda (video on demand).

Estas fórmulas de financiación han sido adecuadas, o cuando menos válidas, en un contexto de emisión lineal, con presencia limitada en el mercado de cadenas generalistas y temáticas. Sin embargo, se vislumbra un nuevo horizonte mediático impulsado por el all digital, aún en fase de consolidación, modificado por elementos estructurales y coyunturales en el que la explosión de la oferta es una realidad (De Matteis Tortora, 2006). En este nuevo ecosistema digital de audiencias fragmentadas y consumo singularizado es preciso redefinir los modelos de explotación de los contenidos audiovisuales ${ }^{10}$.

Si bien inicialmente las prospectivas apuntan hacia una probable coexistencia inicial de los modelos de gestión de contenidos lineales (ofertas cerradas, push) y no lineales (ofertas abiertas, pull), progresivamente los primeros irán perdiendo su centralidad en favor de los segundos. De ahí que sea obligado reflexionar sobre nuevos modelos de negocio y sistemas de financiación más convenientes, aptos para este escenario en permanente evolución. Efectivamente, el nuevo ecosistema digital propicia la consolidación de nuevos modelos de negocio en el entorno multipantalla, al tiempo que demanda la necesidad de dotar a estos modelos de las fórmulas de financiación adecuadas a este singular escenario en el que coexistirán las fórmulas de consumo de contenidos gratuitas y de pago.

Parece evidente que publicidad continuará ocupando un lugar destacado, en cuanto fuente de financiación, dada su versatilidad. Tradicionalmente ha sabido ir adaptándose a los cambios que históricamente ha ido demandando la evolución mediática. Pero lo más destacado es que, en ese continuo estado de adaptación, la publicidad se ha preocupado constantemente por encontrar nuevas maneras de relacionarse con el medio de comunicación que la acoge (Prado y otros, 2007).

En estos momentos en que nos adentramos en la era digital, la publicidad debe adecuar su estrategia comunicativa desarrollando diversos imperativos como la interactividad o la innovación y experimentación en formatos ${ }^{11}$ y narrativas, condicionados por el nuevo estatus activo/selectivo que pasa a ostentar el usuario/ consumidor de contenidos audiovisuales en el ecosistema digital (Roel, 2008).

\footnotetext{
${ }^{10} \mathrm{Creemos}$ interesante destacar el proyecto 3.0 inaugurado el 12 de mayo de 2009 e impulsado por el grupo Antena 3. Se trata de un proyecto de gestión de contenidos multiplataforma innovador que opta por la emisión transversal de contenidos con productos específicos para cada pantalla. "La ficción de 'Globomedia' podrá verse en la televisión analógica con un formato tradicional; en 'Neox', con un 'diario de rodaje' en la que los actores y el director comenten las escenas; en Internet, donde los usuarios intercambiarán sus opiniones 'on line' que se verán automáticamente en la pantalla de 'Nova'. Y, en directo, a través del móvil. Asimismo, se debe mencionar la puesta en marcha del sistema de publicidad de 'pauta única', en la que se comercializan las inserciones de forma conjunta en sus tres canales". Cfr. http://www.elmundo.es/elmundo/2009/05/07/comunicacion/1241714588.html [Consulta: 8 mayo 2009].

${ }^{11}$ Sobre estas cuestiones, consideramos interesante consultar el artículo de Prado y otros (2007) en el que se hace una propuesta sobre fórmulas publicitarias interactivas.
} 
Pero además de la financiación a través de publicidad, en la actualidad existen otros modelos de explotación de contenidos vinculados exclusivamente a la televisión digital de pago a través de satélite, de cable o e Internet (Asimelec, 2008):

1. La suscripción por acceso a contenidos que generalmente se estructuran en paquetes básicos de canales generalistas y temáticos por los que el usuario paga la suscripción mínima junto con paquetes de canales Premium que requieren el abono de una cantidad suplementaria.

2. El pago por visión a través del cual el usuario paga por la visualización de contenidos que se emiten con un horario prefijado. Los contenidos más usuales para esta modalidad son los eventos deportivos y las películas.

3. Y el vídeo bajo demanda o vídeo club on line que permite al usuario el acceso al contenido que desee visualizar sin restricciones horarias. El contenido puede descargarse en el decodificador o bien ser reproducido en streaming ${ }^{12}$.

Por su parte, respecto a los servicios de televisión a través del móvil, el modelo de negocio más utilizado en el territorio español es el de la suscripción por acceso a un paquete de canales. Pero ¿que sucederá con los modelos de explotación de contenidos no lineales?

Los nuevos modelos podrían incluir la reserva temporal de una parte de la pantalla para publicidad, la publicidad personalizada previa a la visión del contenido, flashes intermedios con publicidad, product placement y esponsorización (con advertencia), o incluso la producción directa de contenidos por parte del anunciante (Gaptel, 2006). Además, es preciso tener presentes las potencialidades de la publicidad interactiva, que a priori cuenta con el interés de quien interactúa y que ofrece al usuario un valor añadido. O el posible desarrollo de la publicidad virtual que permite la inserción de objetos o logotipos durante la emisión de un determinado contenido, si bien plantea el inconveniente de imposibilitar la separación de contenidos comerciales y no comerciales.

Se trataría por tanto de introducir fórmulas publicitarias mínimamente intrusivas, que no impidan al usuario el seguimiento del contenido seleccionado y cuyos parámetros resulten adecuados al nuevo entorno digital no lineal.

\section{LA APUESTA POR LA CALIDAD AUNQUE YA NO SEA POSIBLE MANTENER POR MUCHO TIEMPO EL CONSUMO MASIVO IMPULSADO POR LAS OFERTAS LINEALES}

Tal y como se mencionaba anteriormente, es preciso definir las fórmulas de financiación adecuadas que permitan sustentar los nuevos modelos de negocio

\footnotetext{
${ }^{12}$ Tecnología que permite visualizar el contenido sin necesidad de descargarlo en el dispositivo.
} 
capaces de producir y distribuir contenidos audiovisuales. Ahora, el desafío para el sector estriba en que los contenidos que circulen por los nuevos medios sean capaces de adecuarse a los parámetros de calidad deseables. Si bien no existe consenso sobre el concepto de calidad, sí existe -tal y como indica SánchezTabernero (2008) - una percepción coincidente sobre las causas de ese fenómeno, basadas más en impresiones que en evidencias empíricas. Por ello, desde nuestro punto de vista, es altamente recomendable fomentar foros de reflexión que cuenten con la presencia de académicos, empresarios de la comunicación, profesionales y sectores sociales representativos en los que se logre consensuar los parámetros de calidad exigibles a los productos audiovisuales.

Efectivamente, la financiación puede considerarse unánimemente uno de los factores más importantes que inciden directamente sobre el sustento de la calidad en televisión (Gutiérrez Gea, 2000). De ahí la necesidad de encontrar, tal y como se ha apuntado, modelos de gestión rentables tanto en entornos pull como push presentes en la nueva era televisiva. Sin embargo, se podría aludir a otros parámetros capaces de contribuir activamente a perfilar el contexto en el que ha de fraguarse la calidad en los entornos audiovisuales, obviando la calidad técnica exigible que, ineludiblemente, debe presidir toda la cadena de valor ${ }^{13}$ :

Cabría destacar, en primer lugar la observancia de la legislación vigente en cuanto factor impulsor de la calidad. En el ámbito de la Unión Europea se ha desarrollado un importante marco político ${ }^{14}$ que ha ido renovándose con el transcurso de los años, con objeto de adecuarse a la evolución tecnológica que afecta permanentemente al sector audiovisual.

Las políticas audiovisuales que pretendan operar en sintonía con la calidad deberían, al menos, promover medidas de protección para los menores, fomentar la creación, producción y distribución de productos audiovisuales autóctonos (bienes culturales), apostar por la transmisión de valores socialmente aceptados o delimitar las tipologías de contenidos potencialmente prohibidos y restringidos a fin de establecer medidas de protección hacia el usuario. En este sentido, creemos oportuno destacar la aprobación de la Ley General de la Comunicación

\footnotetext{
${ }^{13}$ Eslabones de la cadena de valor del sector audiovisual: Ideación/Creatividad (elaboración de contenidos; ejecución del programa), Producción (grabación, edición), Empaquetamiento (diseño de programación; adaptación/procesos finales), Distribución (difusión; transporte), Consumo (acceso; dispositivo) (Red.es, 2008: 48).

${ }^{14}$ Consideramos importante destacar la Directiva de Televisión sin Fronteras 89/552/CCE (DO L 298, 17/10/1989), documento de referencia inicialmente aprobado en octubre de 1989 y modificado en 1997 (Directiva 97/36/CE; DO L 202, 30/7/1997) que traza las directrices sobre contenidos audiovisuales en los países miembros de la UE. En España está en vigor desde 1994 (Ley 25/1994 de 12 de julio, BOE, 13/7/1994). En diciembre de 20007 ha sido nuevamente modificada (Directiva 2007/65/ CE de Servicios de Medios Audiovisuales Sin Fronteras, de 11 de diciembre de 2007, (DO L 332, 18/12/2007)) con objeto de adecuarse a las nuevas necesidades tecnológicas que afectan a los contenidos audiovisuales. En este documento se establecen normas mínimas sobre protección de menores o sobre inserción de publicidad, al tiempo que se pretende fomentar el pluralismo de los medios de comunicación y la diversidad cultural. Se puede ampliar la información sobre la Política Audiovisual en la Unión Europea a través del siguiente enlace http://europa.eu/pol/av/index_es.htm.
} 
Audiovisual (Ley 7/2010 de 31 de marzo; BOE 1/4/2010) que además de ser un instrumento unificador de la normativa sobre televisión en España, contempla en gran medida los elementos anteriormente mencionados.

Pero además del establecimiento de un marco político regulador acorde con las nuevas circunstancias del entorno digital, es igualmente importante el impulso de iniciativas, consensuadas entre los actores implicados, que diseñen, implanten y consoliden la autorregulación sectorial, que incluyan mecanismos de control social y sanciones en caso de incumplimiento y que complementen las disposiciones legislativas establecidas por los Estados.

Asimismo, es deseable que un ecosistema audiovisual de calidad apueste por la creación, producción y distribución de contenidos diversificados, en permanente sintonía con las tendencias sociales pero, a la vez, respetuosos con el público, sabiendo que en el nuevo contexto de desarrollo del sector, caracterizado por la explosión de la oferta y la fragmentación de la audiencia, todo contenido interesa a alguien ${ }^{15}$ (Gaptel, 2006).

Y finalmente, se debe estimular la innovación y experimentación permanente de formatos, que deben adecuarse a la nueva realidad tecnológica interactiva, a la distribución multipantalla -que puede ser una vía para optimizar la rentabilidad de los productos audiovisuales- y a su adecuación a los parámetros físicos de las distintas pantallas.

\section{LA PROTECCIÓN DE LOS MENORES EN EL NUEVO ESCENARIO AUDIOVISUAL}

Otro de los desafíos que desde nuestro punto de vista debería afrontar el sector hace referencia a la protección de menores frente a determinados contenidos que podrían interferir negativamente en su desarrollo. A lo largo de los últimos años, en el contexto de emisión de contenidos de los modelos de programación convencionales, ha habido diversas iniciativas políticas que corroboran esta afirmación.

Así, la Directiva de Televisión sin Fronteras (DO L 298, de 17/10/1989), además de garantizar la libre difusión y recepción de las emisiones televisivas en el ámbito comunitario, de fomentar las producción y programación de los productos audiovisuales europeos (apostando, en definitiva, por la promoción de la industria autóctona) o de regular y limitar las emisiones publicitarias, intenta proteger a los menores -el sector de la población potencialmente más vulnerable- respecto a determinados contenidos audiovisuales (pornografía, violencia gratuita, publicidad inadecuada, etc.) emitidos en franjas horarias potencialmente infantiles.

\footnotetext{
${ }^{15} \mathrm{En}$ el modelo pull, los contenidos refuerzan su valor en la singularidad de su oferta rompiendo con la tradicional valorización de los contenidos en cuanto eslabón de la programación en su conjunto.
} 
Sobre la protección de menores, debemos mencionar algunas iniciativas vinculantes impulsadas en España tras la entrada en vigor de la Directiva, en respuesta a las directrices marcadas desde Bruselas. En primer lugar, el establecimiento del horario de protección al menor comprendido entre las 6 y las 22 horas y de las franjas de protección reforzada entre las 8 y las 9 horas y entre las 17 y las 20 horas los días laborables, mientras que los fines de semana y días festivos será de 9 a 12 horas. De manera que los contenidos calificados como recomendados para mayores de 13 años deberán emitirse fuera de esos horarios "verdes" (Ley 22/1999 de 7 de junio, BOE, 8/6/1999; Ley 7/2010 de 31 de marzo, BOE, 1/4/2010). Y, en respuesta a lo anterior, la aprobación del Código de señalización orientativa sobre la idoneidad de la programación de televisión (Real Decreto 410/2002 de 3 de mayo, BOE, 23/5/2002) ${ }^{16}$.

En espera de la creación en España del Consejo Estatal de Medios Audiovisuales (CEMA $)^{17}$, una autoridad reguladora del sector audiovisual con amplias competencias e independiente de los poderes fácticos ${ }^{18}$, previsto en la ya mencionada Ley General de la Comunicación Audiovisual (Ley 7/2010 de 31 de marzo, BOE 1/4/2010), las competencias sobre control de contenidos televisivos a lo largo de esta última etapa de gobierno socialista, han recaído sobre el Ministerio de Industria, Turismo y Comercio.

Por otro lado, debemos señalar la existencia en España del Código de autorregulación sobre contenidos televisivos e infancia (www.tvinfancia.es). Este código se firma el 9 de diciembre de $2004^{19}$ con el apoyo del gobierno socialista y de las principales cadenas de televisión españolas. En la actualidad continúa en vigor aunque su cumplimiento no resulta plenamente satisfactorio ${ }^{20}$.

\footnotetext{
${ }^{16}$ Este código de señalización establece que los programas especialmente recomendados para la infancia podrán incluir un símbolo de color verde. Los dirigidos a todos los públicos no llevarán indicativos. Los programas no recomendados para menores de 7 años deberán incluir un símbolo de color amarillo en el que se insertará la cifra 7. Aquellos programas no recomendados para menores de 13 años incluirán un símbolo -también de color amarillo- pero con la cifra 13. Los programas para mayores de 18 años deberán incluir un símbolo rojo con la cifra 18. Y, finalmente, los programas clasificados $\mathrm{X}$ se identificarán con el símbolo rojo pero incluyendo la letra $\mathrm{X}$. Los programas obligados a identificarse con el símbolo rojo deberán realizar simultáneamente una advertencia acústica de un segundo de duración.

${ }^{17}$ En el momento de cerrar la última versión de este artículo, la creación del CEMA aún no se había producido.

${ }^{18} \mathrm{La}$ necesidad de crear una alta autoridad del audiovisual en España con amplias competencias se recoge ya en las Conclusiones publicadas por la Comisión Especial del Senado sobre contenidos televisivos en el año 1995 (BOCG, Senado. V Legislatura, $n^{\circ} 262$, pp. 77-79). A lo largo de los últimos años tan solo hemos podido constatar la existencia de altas autoridades del audiovisual a nivel autonómico (Cataluña, Navarra y Andalucía).

${ }^{19} \mathrm{En}$ marzo de 1993 se había firmado otro código semejante impulsado por el entonces ministro de Educación Alfredo Pérez Rubalcaba y consensuado entre todas las cadenas de televisión existentes en aquella fecha. Fue un fracaso por sus reiterados incumplimientos. Con el tiempo, ha caído en el olvido.

${ }^{20}$ Ver IX Informe ATR-Villanueva de seguimiento del Código de Autorregulación en horario de protección reforzada de 17 de junio de 2009 accesible en www.atr.org.
} 
De todo lo expuesto se puede deducir que efectivamente existen iniciativas políticas reguladoras y autorreguladoras dirigidas a la protección de los menores aunque los actores implicados en el sector no siempre las acaten.

Pero ¿qué sucederá cuando los modelos de gestión de contenidos mayoritarios no sean lineales? ¿Cómo se podría proteger al menor cuando ya no sea posible establecer horarios protegidos ("horarios verdes")?

La Comisión Europea, consciente de ello ha incluido en la nueva Directiva de Servicios Audiovisuales Sin Fronteras ${ }^{21}$ diversas propuestas con las que pretende proteger a los más jóvenes. Tal y como se indica en los Considerandos iniciales ${ }^{22}$ se debe

equilibrar cuidadosamente las medidas para proteger a los menores y la dignidad humana con el derecho fundamental a la libertad de expresión consagrado en la Carta de los Derechos Fundamentales de la Unión Europea. La finalidad de este tipo de medidas, tales como el uso de códigos PIN (números de identificación personal), de sistemas de filtrado o de identificación, debe ser, pues, asegurar un adecuado nivel de protección del menor y de la dignidad humana, en especial en relación con los servicios de comunicación audiovisual a petición [...].

La protección de menores en el nuevo entorno digital debe contar con un marco político que lo garantice y con el apoyo de los todos los actores implicados en el desarrollo de la cadena de valor del sector pero, ineludiblemente, debe contar con la actitud activa de padres y tutores que son, en definitiva, los máximos responsables del consumo audiovisual de los menores que tienen a su cargo. Dado que en los entornos no lineales ya no será posible el establecimiento de los denominados "horarios verdes", la ecología audiovisual pasa por estrechar la colaboración entre todos los actores implicados en el proceso comunicativo audiovisual con objeto de garantizar también la protección de los menores.

\section{CONCLUSIONES}

En esta etapa de consolidación del ecosistema digital, de explosión de la oferta y de interactividad, la televisión afronta una nueva edad -la Postelevisióninmersa en importantes transformaciones que afectan a toda la cadena de valor e intentando dar respuesta a diversos desafíos directamente vinculados a la

\footnotetext{
${ }^{21}$ Directiva 2007/65/CE del Parlamento Europeo y del Consejo, de 11 de diciembre de 2007, por la que se modifica la Directiva 89/552/CEE del Consejo sobre la coordinación de determinadas disposiciones legales, reglamentarias y administrativas de los Estados miembros relativas al ejercicio de actividades de radiodifusión televisiva (Diario Oficial $n^{\circ}$ L 332 de 18/12/2007, pp. 0027-0045).

${ }^{22}$ Ver Considerandos $n^{\circ} 45$ y 46. Directiva 2007/65/CE del Parlamento Europeo y del Consejo, de 11 de diciembre de 2007, por la que se modifica la Directiva 89/552/CEE del Consejo sobre la coordinación de determinadas disposiciones legales, reglamentarias y administrativas de los Estados miembros relativas al ejercicio de actividades de radiodifusión televisiva (Diario Oficial $n^{\circ} L 332$ de 18/12/2007).
} 
evolución del paisaje audiovisual. La consecución de los retos que se proponen en el presente artículo requiere la actuación armónica y sincrónica de todos los actores implicados: políticos, empresarios de la comunicación, profesionales y usuarios.

La democratización digital, la producción de contenidos audiovisuales multiplataforma junto con la experimentación en narrativas interactivas, la delimitación de los modelos de negocio, la búsqueda de estándares de calidad consensuados o las nuevas medidas dirigidas a garantizar la protección de los menores se perfilan como elementos imprescindibles y determinantes en la progresiva construcción de esta nueva edad televisiva. Por todo ello, parece determinante el impulso de políticas públicas capaces de garantizar el acceso universal a la cultura digital, que operen en sintonía con parámetros de calidad en las líneas señaladas y que contemplen medidas dirigidas a garantizar la protección de los menores y de los usuarios en general o la protección de la cultura autóctona.

Es éste el contexto en el que debe desarrollarse el mercado de los contenidos audiovisuales (necesariamente innovador y creativo), un sector estratégico en constante expansión que deberá ser capaz de satisfacer la creciente demanda así como adecuar sus modelos de negocio a la nueva realidad digital sabiendo que la rentabilidad pasa por la combinación de modelos gratuitos sustentados por publicidad (convencional, interactiva) y los modelos de explotación de pago (suscripción, pago por visión, bonos recargables, etc.).

Hoy por hoy, la consolidación del ecosistema digital del que la televisión forma parte, se debate entre determinadas certezas resueltas en el presente e incertidumbres que se irán despejando en un futuro más o menos próximo.

\section{REFERENCIAS BIBLIOGRÁFICAS}

ARROYO, M.; y ROEL, M. (2006): Los medios de comunicación en la democracia (19822005). Prensa, radio, televisión, Internet y grupos de comunicación. Madrid: Fragua.

ASIMELEC y OTROS (2008): Informe de la Industria de contenidos digitales 2008. Madrid: ASIMELEC y Dirección General de Sociedad de la Información, (Secretaría de Estado de Telecomunicaciones y para la Sociedad de la Información, Ministerio de Industria, Turismo y Comercio).

BOLETÍN OFICIAL DE LAS CORTES GENERALES (BOCG).

BOLETÍN OFICIAL DEL ESTADO (BOE).

BUSTAMANTE, E.: Lecciones de un laboratorio peculiar. La televisión digital y sus tortuosos caminos. En BUSTAMANTE, E. (coord.) (2007): Cultura y comunicación para el siglo XXI. Diagnóstico y políticas públicas. La Laguna (Tenerife): Ideco, pp. 135-160.

CARUSO, M. P. (coord.) (2006): L'audiovisivo e la svolta digitale. Soveria Mannelli (Italia): Rubbettino. 
DE MATTEIS TORTORA, G.: Le nuove forme di offerta e consumo di tv digitale all'interno del mercato della convergenza. En CARUSO, M. P. (coord.) (2006): L'audiovisivo e la svolta digitale. Soveria Mannelli (Italia): Rubbettino, pp. 61-74.

DIARIO OFICIAL DE LAS COMUNIDADES EUROPEAS.

FRANCÉS I DOMĖNEC, M. (coord.) (2009): Hacia un nuevo modelo televisivo. Contenidos para la televisión digital. Barcelona: Gedisa.

FUNDACIÓN ORANGE (2008): eEspaña 2008. Informe anual sobre el desarrollo de la Sociedad de la Información en España. Madrid: Fundación Orange.

FUNDACIÓN TELEFÓNICA (2009): Informe de la Sociedad de la Información. Madrid: Ariel [en línea: http://e-libros.fundacion.telefonica.com/sie09/aplicacion sie.html Consulta 7 junio 2010].

GAPTEL (2006): Contenidos digitales. Nuevos modelos de distribución on line. Grupo de Análisis y Prospectiva del Sector de las Telecomunicaciones, Madrid: Red.es [en línea: http:// observatorio.red.es/gaptel/archivos/ContenidosDigitales final.pdf, Consulta: 6 marzo 2009].

GARCÍA, A.; VINADER, R.: "Televisión tradicional y televisión móvil. Estrategias para contenidos televisivos en movilidad", Telos $n^{\circ} 83$ (mayo 2010) [en línea: http:// sociedadinformacion.fundacion.telefonica. com/seccion=1266\&idioma=es ES\&id=20100512 13360001\&activo=6.do , Consulta: 9 junio 2010].

GUTIÉRREZ GEA, C.: "Televisión y calidad: perspectivas de investigación y criterios de evaluación", Zer, no 9 (2000) [en línea: http://www.ehu.es/zer/zer9/9gutierrez.html, Consulta: 18 junio 2009].

IMBERT, G. (2008): El transformismo televisivo. Postelevisión e imaginarios sociales. Madrid: Cátedra.

IMPULSA TDT (2008): Anuario TDT 2008 [en línea: http://www.impulsatdt.es/pdf/impulsatdt-anuario-08.pdf , Consulta 22 junio 2009].

- (2010): TDT Informe final. Madrid: Impulsa TDT.

KÖSTER, C. R.: "Nuevos relatos audiovisuales. Hacia una definición del relato audiovisual interactivo", Telos, n 62 (enero-marzo 2005)

[en línea: http://www.campusred.net/telos/articulocuaderno.asp?idarticulo=4\&rev=62, Consulta 23 marzo 2009].

PISCITELLI, A. (1998): Postelevisión. Ecología de los medios en la Era de Internet. Buenos Aires: Paidós.

POSTIGO GÓMEZ, I.: "Calidad y televisión digital terrestre", Comunicar, n 25 (2005), [en línea: http://dialnet.unirioja.es/servlet/articulo?codigo=2927177, Consulta 18 junio 2009].

PRADO, E.; FRANQUET, R.; RIBES, X.; SOTO, M.; FERNÁNDEZ QUIJADA, D.: "La publicidad televisiva ante el reto de la interactividad", Questiones Publicitarias, $\mathrm{n}^{\circ} 12$ (2007), pp. 13-28.

PRETA, A. (2007): Economia dei contenuti. L'industria dei media e la rivoluzione digitale. Milano (Italia): Vita e Pensiero.

RAMONET, I. (ed.) (2002): La Postelevisión. Multimedia, Internet y globalización económica. Barcelona: Icaria-Antrazyt. 
RED.ES (2008): Libro Blanco de los contenidos digitales en España 2008. Madrid: Red.es [en línea http://www.red.es/publicaciones/articles/id/2660/libro-blanco-contenidos-digitales2008.html, Consulta: 22 junio 2009].

ROSÉS, J. (2009): Interactividad en TDT: 2009, una oportunidad. En Anuario TDT 2008. Madrid: Impulsa TDT, pp. 106-107.

ROEL, M.: La televisión en la democracia española (1982-2005). Dinámicas de los modelos de gestión de contenidos. En ARROYO, M.; y ROEL, M. (2006): Los medios de comunicación en la democracia (1982-2005). Prensa, radio, televisión, Internet y grupos de comunicación. Madrid: Fragua pp. 91-159.

- "Audiovisual digitalization in Spain and Italy: from Neotelevision to Postelevision", Observatorio $\left(O B S^{*}\right)$, Journal 4 (Febrero 2007), Lisboa (Portugal) pp. 95-112.

- "Contenidos audiovisuales en el entorno multipantalla: gestión e innovación", Trípodos Extra 2009, vol. 2 (2009), pp.1071-1080.

SÁNCHEZ-TABERNERO, A. (2008): Los contenidos de los medios de comunicación: calidad, rentabilidad y competencia. Barcelona: Deusto.

SAVONA, S. (coord.) (2006): L'audiovisivo e la svolta digitale. Catanzaro (Italia): Rubbetino.

ZALLO R.: Las políticas culturales territoriales revisadas. En BUSTAMANTE, E. (coord.) (2007): Cultura y comunicación para el siglo XXI. Diagnóstico y políticas públicas. La Laguna (Tenerife): Ideco, pp. 215-257.

\section{Páginas web:}

www.atr.org

www.elmundo.es

www.iabspain.net

www.live.vodafone.es

www.mmaglobal.com

www.red.es

www.rtve.es

www.tvinfancia.es

\section{Breve semblanza biográfica de la autora}

Marta Roel es Profesora de la Universidad de Murcia. Actualmente es coordinadora del Grado en Comunicación Audiovisual y miembro del grupo de investigación e-COM donde participa como investigadora en "Movilizad@s: La telefonía móvil en la S.I. como dispositivo fundamental para la igualdad de oportunidades" (I+D+i 135/06) y "Evolución del medio móvil en España: actores, contenidos, modelos de negocio y percepción de los usuarios" (I+D: CSO2009-07108).

(Recibido el 30-06-2009, aceptado el 14-03-2010) 